\title{
Concurrent topological design of composite structures and the underlying multi-phase materials
}

\author{
D.C. $\mathrm{Da}^{\mathrm{a}, \mathrm{b}}$, X.Y. Cui ${ }^{\mathrm{a}, \mathrm{b}^{*}}$, K. Long ${ }^{\mathrm{c}}$, G.Y. Li $\mathrm{Li}^{\mathrm{a}, \mathrm{b}^{*}}$ \\ ${ }^{a}$ State Key Laboratory of Advanced Design and Manufacturing for Vehicle Body, \\ Hunan University, Changsha, PR China \\ ${ }^{\mathrm{b} J o i n t}$ Center for Intelligent New Energy Vehicle, Shanghai, PR China \\ 'State Key Laboratory for Alternate Electrical Power System with Renewable Energy Sources, \\ North China Electric Power University, Beijing, PR China
}

\begin{abstract}
This paper presents a concurrent topology optimization approach for simultaneous design of composite structures and their periodic material microstructures with three or more phases. The effective properties of multi-phase materials are obtained via homogenization technique which serves as a bridge of the finite element models of the macrostructure and the material microstructure. The base materials of periodic microstructures used in each phase of the macrostructure are divided into several groups and sensitivity analysis are carried out on them one by one. Meanwhile, the sensitivity number at the macrostructure is derived which is coupled with the designed material properties. Then, the composite configurations of material microstructures and macrostructures are inversely optimized concurrently based on the bi-directional evolutionary structural optimization (BESO) algorithm. Several 2D and 3D numerical examples are presented to demonstrate the effectiveness of proposed design approach.
\end{abstract}

Keyword: Multi-material topology optimization; Concurrent design; Inverse homogenization; Bidirectional evolutionary structural optimization (BESO) 


\section{Introduction}

Structural topology optimization aims to find the optimal spatial material distribution within a given design domain for prescribed constraints, objectives, and boundary conditions. The original idea of this technique is to determine which points of the design domain should be material points and which points should remain void (no material) [1]. Over the past decades, topology optimization has undergone an extensive development in both fields of theoretical research and practical application [2]. Various topology optimization methods have been proposed, which include of homogenization approach [3], solid isotropic materials with penalization (SIMP) [4, 5], level-set method (LSM) [6, 7], evolutionary structural optimization (ESO) $[8,9]$ and bi-directional ESO (BESO) method $[10,11]$ etc. These methods have already demonstrated to be the flexible and reliable design tools in various industrial applications. An overview, comparison and critical review of the different topology optimization approaches was given in [12]. Among all exiting approaches for topology optimization, the ESO/BESO methods use an intuitive design strategy for the design of structural topology. The early ESO algorithms cannot recover the materials once it has been removed, however, in practice the materials that has been removed in an early stage might be required later. For this issue, its later version, namely BESO method allows materials to be added and removed simultaneously. It has been demonstrated that the new BESO method is capable of generating reliable and practical topologies for various types of structures with high computational efficiency [13-15].

Topology optimization has not only been applied for the design of monoscale structures, but also for the design of materials. By means of homogenization approach, one may evaluate the effective material properties of the considered microstructure or Representative Unit Cell (RUC) model [16]. The key hypotheses of the homogenization are that the characteristics of macroscopic structures are much larger than that of material microstructures, and the macrostructures are assembled by the RUC periodically. Sigmund $[17,18]$ introduced an inverse homogenization technique 
to tailor materials with prescribed constitutive parameters and elastic properties using the SIMP method. Some studies were also investigated to tailor material microstructures with other extraordinary properties like thermal conductivity [19], permeability [20], and stiffness and conductivity [21]. Similar researches also were addressed using the level set-based methods [22] and the BESO method [23, 24]. Some other works [25-28] also fall into this topic.

With such model for the design of material microstructures, one comes up naturally with ideas of integrate or concurrent designs of both structures and material microstructures. That is to say, the goal of topology optimization is to determine not only the optimal structural topology or material layout at the macrostructural scale, but also the optimal local use of the porous material or composite at the microstructural scale. Rodrigues at al [29] described a hierarchical computational procedure for optimization of material distribution as well as the local material properties of mechanical elements. Coelho at al [30] presented an extension of this hierarchical model for topology optimization to 3D structures. Ferreira et al [31] performed the hierarchical optimization in laminated composite structures. Xia et al [32-34] addressed concurrent design of material and structure within the $\mathrm{FE}^{2}$ nonlinear multi-scale analysis framework and applied lately in [35] a reduced database model [36] to circumvent the intensive computational cost. These works assumed that the materials/composites used in macrostructural construction vary pointwisely and require in general large amount of computational efforts.

The most commonly used strategy is designing a uniform material microstructure or RUC at the microscopic scale for a concurrently changed macrostructure at the macroscopic scale. Via this design strategy, concurrent design has been used for tailoring macrostructure and its material microstructures with minimum systematic mean compliances in $[37,38]$, maximum fundamental frequencies in $[39,40]$, and multi-objective functions, e.g. maximum stiffness and minimum resistance to heat dissipation [41], minimum structural compliance and minimum thermal expansion of the surfaces [42], and minimum structural mean compliance and material thermal conductivity [43]. Concurrent robust design and optimization considering load 
uncertainties was investigated in [44]. Concurrent design of composite macrostructure and cellular microstructure under random excitations was studied in [45]. But there is litter work on the concurrent topological design of composite structures and their multi-phase materials. In comparison with porous materials, composites consist of two or more phase materials are more attractive and advantageous from the perspective of engineering application. Designing new structures that are composed of multi-phase composite has already attracted much attentions, and a review of recent advances on mechanics of multi-functional composite materials and structures has been given in [46]. Based on the concept of topology optimization, Bendsøe and Sigmund [47] proposed a multi-phase material mixture model in SIMP, and such a model has been extended to various topology optimization problems [48-51]. Recently, different topology optimization methods were employed to design multi-phase material structures like level set-based methods [52-55] and the BESO method [56-58].

This paper builds on the earlier work [56] on multiple material design of monoscale structures using the BESO method where the constituent phases are divided into different groups. This model has already been extended for the design of multi-phase material microstructures [58]. The key contribution of this work is to integrate the BESO method, homogenization and multi-phase material interpolation scheme to carry out multiscale topology optimization with the consideration of multi-phase material microstructures at the lower scale, which has been rarely examined in the literature yet according to our best knowledge. Comparing with only two phase materials employed at microscopic scale in [57], we designed the underlying multi-phase material microstructures for both the solid material phase and the compliant material phase of the macroscale structure. The effective constitutive parameters of three or more materials are evaluated by the numerical homogenization analysis $[59,60]$. In addition, we have carried out the concurrent multi-phase topology optimization for both 2D and 3D cases, which makes this work distinguished from existing references. The remainder of this paper is organized as follows: concurrent optimization model of composite macrostructure and its 
multi-phase materials are drawn in Section 2. Section 3 formulates the material interpolation and gives the sensitivity analysis on macro and micro variables. The BESO method and numerical implementation procedure are introduced in Section 4. Section 5 presents several 2D and 3D numerical examples and discussions. Finally, conclusion part is given in Section 6.

\section{Concurrent optimization formulation}

The concurrent topology optimization problem considers a macrostructure composed of multi-phase materials with periodic microstructures illustrated in Fig. 1. Composite formulation is applied to both the macrostructure and material microstructures. The present work considers the macrostructure is composed of two nonzero periodic composites that both have three or more phase materials. Each multi-phase composite serves as a phase in the macrostructure. Then, there are totally three finite element (FE) models which include one macro model for the macrostructure and two micro models for the material RUCs correspondingly. RUC of the periodic multi-phase composite serves as the design domain in each micro models. The optimization objective is to minimize the structural compliance (or maximize the structural stiffness) by finding the optimal macrostructural topology and the optimal designs of the multi-phase composites used in macrostructural construction.

Similar to the classical structural topology optimization, the volume constraint defines the target volume of each phase/composite is employed both in micro and macro models. It is assumed that each composite consists of $n$ kinds of base materials with equal Poisson's rations and Young's moduli are sorted descending, i.e. $E_{1}^{1}>E_{2}^{1} \ldots>E_{n}^{1}$ and $E_{1}^{2}>E_{2}^{2} \ldots>E_{n}^{2}$, where the superscripts indicate the number of micro model and the subscripts are the number of base materials in each micro model. Such a concurrent topology optimization problem for compliance minimum can be formulated as following:

Find: $X=\left\{x_{m}^{m a c}, x_{i j}^{m i c, 1}, x_{i j}^{m i c, 2}\right\}$ 
Subject to $: V_{1}^{m a c}-\sum_{m=1}^{M} V_{m} x_{m}^{m a c}=0$

$\sum_{i=1}^{N} V_{i} x_{i j}^{\mathrm{mic}, 1}-V_{j *}^{\mathrm{mic}, 1}-\sum_{i=1}^{j-1} V_{i *}^{m i c, 1}=0 \quad(j=1,2, \ldots \ldots, n)$

$\sum_{i=1}^{N} V_{i} x_{i j}^{\mathrm{mic}, 2}-V_{j *}^{\mathrm{mi} c, 2}-\sum_{i=1}^{j-1} V_{i *}^{m i c, 2}=0(j=1,2, \ldots \ldots, n)$

$x_{m}^{m a c}=x_{\min }$ or $1 ; x_{i j}^{m i c, 1}=x_{\min }$ or $1 ; x_{i j}^{m i c, 2}=x_{\min }$ or 1

where $\mathbf{U}$ and $\mathbf{F}$ are the displacement vector and external force vector of the macrostructure, respectively. $\mathrm{M}$ and $\mathrm{N}$ are the total number of finite elements in macro and micro FE models, respectively. The design variable $X$ consists of three subsets: macro design variable $x_{m}^{m a c}$ represents the layout of two composites in the macrostructure; micro design variables $x_{i j}^{m i c, 1}$ and $x_{i j}^{m i c, 2}$ represent the layout of base materials in micro models 1 and 2, respectively. Note that the micro design variable $x_{i j}^{m i c, 1}$ represents the microstructure for macro elements $x_{m}^{m a c}=1$, and $x_{i j}^{m i c, 2}$ for macro elements $x_{m}^{m a c}=x_{\min } . x_{\min }$ is a small predetermined value, e.g. 0.001 . In the following, the design variables $x_{i j}^{\mathrm{mic}, 1}$ and $x_{i j}^{m i c, 2}$ will be consistently denoted as $x_{i j}^{m i c}$ because of the similarity. Therefore, $x_{i j}^{m i c}$ indicates the relative density of the $i$-th element for the $j$-th base material in micro models can be expressed by:

$x_{i j}^{\min }=\left\{\begin{array}{cl}1 & \text { for } E \geq E_{j} \\ x_{\min } & \text { for } E \leq E_{j+1}\end{array}\right.$

where $E$ is the Young's modulus of the $i$-th element. $x_{i j}^{\min }=1$ is adopted when the element is filled with the base material $j$ or the base materials with larger Young's modulus than $j$, and $x_{i j}^{\min , 1}$ is equal to $x_{\min }$ otherwise. Therefore, the term $\sum_{i=1}^{N} V_{i} x_{i j}^{\mathrm{mic}, 1}$ in Eq. (4) represents total volumes of the $j$-th base material and stiffer 
base materials $(1, \ldots, j-1)$ in the first micro model, and the prescribed volume of $j$-th base material is denoted as $V_{j^{*}}^{\text {mic,1 }}$ which can be defined by designers. Similarly, $\sum_{i=1}^{N} V_{i} x_{i j}^{\mathrm{mic}, 2}$ and $V_{j^{*}}^{\mathrm{mic}, 2}$ are the corresponding terms in the second micro model. $V_{i}$ denotes the volume of the $i$-th element in the micro models; $V_{m}$ denotes the volume of the $m$-th macrostructural element and $V_{1}^{\text {mac }}$ is the prescribed volume of the first phase $\left(x_{m}^{m a c}=1\right)$ in the macro model.

\section{Material interpolation scheme and sensitivity analysis}

\subsection{Material interpolation scheme}

The material interpolation scheme is the most essential part to perform the sensitivity analysis on both the micro and macro models, and a SIMP approach is employed here. For three or more phases material design problem in the micro models, it is straightforward to interpolate the material properties between two neighboring phases (i.e. $E_{j}$ and $\left.E_{j+1}\right)[45,54]$, as

$E\left(x_{i j}^{m i c}\right)=\left(x_{i j}^{m i c}\right)^{P} E_{j}+\left[1-\left(x_{i j}^{m i c}\right)^{P}\right] E_{j+1}$

where $P$ is the penalization exponent which is equal to 3 throughout this paper.

Under this scheme, the elasticity matrix $\mathbf{D}$ in the micro models can be expressed as

$\mathbf{D}\left(x_{i j}^{m i c}\right)=\left(x_{i j}^{m i c}\right)^{P} \mathbf{D}_{j}+\left(1-x_{i j}^{m i c}\right) \mathbf{D}_{j+1}$

in which $\mathbf{D}_{j}$ and $\mathbf{D}_{j+1}$ are respectively the elasticity matrix of the base materials $j$ and $j+1$. As for the macro model, the $m$-th element elasticity matrix can be expressed as:

$\mathbf{D}^{m a c}\left(x_{m}^{m a c}\right)=\left(x_{m}^{m a c}\right)^{P} \mathbf{D}^{H, 1}+\left[1-\left(x_{m}^{m a c}\right)^{P}\right] \mathbf{D}^{H, 2}$

in which $\mathbf{D}^{H, 1}$ and $\mathbf{D}^{H, 2}$ are the effective elasticity matrices computed by the 
homogenization implementation of the micro models 1 and 2 respectively. Based on the classical homogenization method, $\mathbf{D}^{H, 1}$ and $\mathbf{D}^{H, 2}$ can be respectively expressed as

$\mathbf{D}^{H, 1}=\frac{1}{|Y|} \int_{Y} \mathbf{D}^{1}(\mathbf{I}-\mathbf{b u}) d Y$

$\mathbf{D}^{H, 2}=\frac{1}{|Y|} \int_{Y} \mathbf{D}^{2}(\mathbf{I}-\mathbf{b u}) d Y$

where $|Y|$ is the total volume of RUC in the micro models; I is the identity matrix and $\mathbf{b}$ is the strain-displacement matrix. $\quad \mathbf{D}^{1}$ and $\mathbf{D}^{2}$ are the elasticity matrices at a point with relative density $x_{i j}^{m i c, 1}$ and $x_{i j}^{m i c, 2}$ in Eq. (9), respectively. $\mathbf{u}$ is the displacement fields caused by imposing uniform strain fields, for instance, $\{1,0,0\}^{T}$, $\{0,1,0\}^{T},\{0,0,1\}^{T}$ in 2D problem.

\subsection{Sensitivity analysis}

In order to calculate the macrostructural static response and conduct the sensitivity analysis, the FE analysis also should be performed for macrostructures at the macro model. In the macro FE model, the structural compliance can be obtained using Eq. (2), and the sensitivity of the compliance with respect to the macro design variable, $x_{m}^{m a c}$, can be written as

$\alpha_{m}^{m a c}=\frac{\partial C}{\partial x_{m}^{m a c}}=-\mathbf{U}^{T} \frac{\partial \mathbf{K}}{\partial x_{m}^{m a c}} \mathbf{U}=-P\left(x_{m}^{m a c}\right)^{P-1}\left(\mathbf{U}_{m}^{T} \mathbf{K}_{m}^{1} \mathbf{U}_{m}-\mathbf{U}_{m}^{T} \mathbf{K}_{m}^{2} \mathbf{U}_{m}\right)$

where $\mathbf{U}_{m}$ is the displacement vector of the $m$-th element in the macro model. $\mathbf{K}_{m}^{1}$ and $\mathbf{K}_{m}^{2}$ are the $m$-th element stiffness matrices calculated from using $\mathbf{D}^{H, 1}$ and $\mathbf{D}^{H, 2}$ respectively, which can be expressed as

$\mathbf{K}_{m}^{1}=\int_{V_{m}} \mathbf{B}^{T} \mathbf{D}^{H, 1} \mathbf{B} d V_{m}$

$\mathbf{K}_{m}^{2}=\int_{V_{m}} \mathbf{B}^{T} \mathbf{D}^{H, 2} \mathbf{B} d V_{m}$

where $\mathbf{B}$ is the strain-displacement matrix. 
As for the micro models, the design variables $x_{i j}^{m i c, 1}$ and $x_{i j}^{m i c, 2}$ are respectively related to all first phase $\left(x_{m}^{m a c}=1\right)$ and second phase $\left(x_{m}^{m a c}=x_{\min }\right)$ in the macro model. Then, the sensitivity numbers of the objective function on the micro design variables $x_{i j}^{m i c, 1}$ and $x_{i j}^{m i c, 2}$ are respectively equal to the summation of derivatives of the objective function over all first phase $\left(x_{m}^{m a c}=1\right)$ and second phase $\left(x_{m}^{m a c}=x_{\min }\right)$ in the macro model as

$$
\begin{aligned}
& \alpha_{i j}^{m i c, 1}=\frac{\partial C}{\partial x_{i j}^{m i c, 1}}=-\sum_{m=1}^{M}\left(x_{m}^{m a c}\right)^{P} \mathbf{U}_{m}^{T} \frac{\partial \mathbf{K}_{m}^{1}}{\partial x_{i j}^{m i c, 1}} \mathbf{U}_{m}=-\sum_{m=1}^{M} x_{m}^{P} \mathbf{U}_{m}^{T} \int_{V_{m}} \mathbf{B}^{T} \frac{\partial \mathbf{D}^{H, 1}}{\partial x_{i j}^{m i c, 1}} \mathbf{B} d V_{m} \mathbf{U}_{m} \\
& \alpha_{i j}^{m i c, 2}=\frac{\partial C}{\partial x_{i j}^{m i c, 2}}=-\sum_{m=1}^{M}\left(1-x_{m}^{m a c}\right)^{P} \mathbf{U}_{m}^{T} \frac{\partial \mathbf{K}_{m}^{2}}{\partial x_{i j}^{m i c, 2}} \mathbf{U}_{m}=-\sum_{m=1}^{M}\left(1-x_{m}\right)^{P} \mathbf{U}_{m}^{T} \int_{V_{m}} \mathbf{B}^{T} \frac{\partial \mathbf{D}^{H, 2}}{\partial x_{i j}^{m i c, 2}} \mathbf{B} d V_{m} \mathbf{U}_{m}
\end{aligned}
$$

With the help of material interpolation scheme in Eqs. (8) and (9), the derivative of homogenized elasticity matrices with respect to the design variables $x_{i j}^{m i c, 1}$ and $x_{i j}^{m i c, 2}$ can be expressed as following using the adjoint variable method

$$
\frac{\partial \mathbf{D}^{H, 1}}{\partial x_{i j}^{m i c, 1}}=\frac{1}{|Y|} \int_{Y}(\mathbf{I}-\mathbf{b u}) \frac{\partial D^{1}}{x_{i j}^{m i c, 1}}(\mathbf{I}-\mathbf{b u}) d Y=\frac{P\left(x_{i j}^{m i c, 1}\right)^{P-1}}{|Y|} \int_{Y}(\mathbf{I}-\mathbf{b u})\left(\mathbf{D}_{j}^{1}-\mathbf{D}_{j-1}{ }_{j-1}\right)(\mathbf{I}-\mathbf{b u}) d Y
$$

$$
\frac{\partial \mathbf{D}^{H, 2}}{\partial x_{i j}^{m i c, 2}}=\frac{1}{|Y|} \int_{Y}(\mathbf{I}-\mathbf{b u}) \frac{\partial \mathbf{D}^{2}}{x_{i j}^{m i c, 2}}(\mathbf{I}-\mathbf{b u}) d Y=\frac{P\left(x_{i j}^{m i c, 2}\right)^{P-1}}{|Y|} \int_{Y}(\mathbf{I}-\mathbf{b u})\left(\mathbf{D}_{j}^{2}-\mathbf{D}_{j-1}^{2}\right)(\mathbf{I}-\mathbf{b u}) d Y
$$

From the above analysis, the homogenized elasticity matrices calculated from the micro models are integrated to the macrostructural FE analysis. In addition, the sensitivity numbers of elements in the micro models consider the displacement field of the macrostructure. In other words, all three FE analysis models are interacting to each other and the topological design of composite macrostructure and multi-phase material microstructures are executed concurrently.

\section{BESO method and numerical implementation procedure}




\subsection{Sensitivity numbers}

The sensitivity information in the BESO method can be used to represent the relative ranking of the sensitivity of an individual element, thus, the sensitivity number of the $m$-th element in the macro model for minimizing the compliance can be rewritten as

$\alpha_{m}^{m a c}=-\frac{1}{P} \frac{\partial C}{\partial x_{m}^{m a c}}$

Similarly, the sensitivity numbers in the micro models can be rewritten as

$\alpha_{i j}^{m i c, 1}=-\frac{1}{P} \frac{\partial C}{\partial x_{i j}^{m i c, 1}}$

$\alpha_{i j}^{m i c, 2}=-\frac{1}{P} \frac{\partial C}{\partial x_{i j}^{m i c, 2}}$

Using Eqs. (13), (16), (17), (18) and (19), the sensitivity number $\alpha_{m}^{m a c}$ at the macro model and sensitivity numbers $\alpha_{i j}^{m i c, 1}$ and $\alpha_{i j}^{m i c, 2}$ at the micro models can be easily obtained. In addition, since all design variables are either 1 or $x_{\min }$, a simple scheme can be devised to update the design variables. For instance, elemental relative densities in the macro model may change from $\alpha_{m}^{m a c}=1$ to $\alpha_{m}^{m a c}=x_{\min }$ for holding lower sensitivity numbers and from $\alpha_{m}^{m a c}=x_{\min }$ to $\alpha_{m}^{m a c}=1$ for holding higher sensitivity numbers.

\subsection{Variables updating}

With the above updating scheme, the volumes of two phases in the macro model are gradually changed according the sensitivity number $\alpha_{m}^{m a c}$ and the target volume of first or second phase for the next iteration. The target volume of first phase in the macro mode for the next iteration can be defined as

$\left(V^{m a c}\right)^{k+1}=\left(V^{m a c}\right)^{k}(1 \pm E R)$

where ER is the evolution rate which can be determined by designers. When the 
current volume of first phase in the macro model, $\left(V^{m a c}\right)^{k}$, is larger than the prescribed volume $V_{1}^{\text {mac }}$, a minus sign is applied in Eq. (23). If the obtained $\left(V^{m a c}\right)^{k+1}$ is less than $V_{1}^{m a c}$, then $\left(V^{m a c}\right)^{k+1}$ is set to $V_{1}^{m a c}$. Similarly, the plus sign is applied in Eq. (23) when $\left(V^{\text {mac }}\right)^{k}$ is less than the target volume $V_{1}^{\text {mac }}$. If the obtained $\left(V^{m a c}\right)^{k+1}$ is larger than $V_{1}^{m a c}$, then $\left(V^{m a c}\right)^{k+1}$ is set to $V_{1}^{m a c}$.

At the micro models, firstly, the sensitivity numbers of design variables $\alpha_{i j}^{m i c, 1}$ and $\alpha_{i j}^{m i c, 2}$ are uniformly denoted as $\alpha_{i j}^{m i c}$ for convenience in the following. Note that the sensitivity number $\alpha_{i j}^{m i c}$ is defined in the whole area of the RUC even though it is only used for interchanging base materials $j$ and $j+1$. Consider $n$ ordered base materials used in each micro model, there are $n-1$ ordered groups of materials and $n-1$ groups of sensitivity numbers to make adjustments between neighboring base materials. Take $n=3$ as an example, the value of $j$ can take 1 and 2 which means there are two groups of sensitivity numbers in each micro model named as $\alpha_{i 1}^{m i c}$ and $\alpha_{i 2}^{m i c}$. The optimization procedure of topological design of multi-phase materials' microstructures starts from a nearly full design with base material 1 , and small amounts of base material 2. After the sensitivity analysis with respect to the design variable $x_{i 1}^{\text {mic }}$, transitions of base materials 1 and 2 in the micro models are executed according to the sensitivity number $\alpha_{i 1}^{\text {mic }}$ and the target volume of base material 2 for the next iteration. The target volume of base material 2 for the next iteration can be defined as

$\left(V_{2}^{\mathrm{mic}}\right)^{k+1}=\min \left(\left(V_{2}^{\mathrm{mic}}\right)^{k}(1+E R), V_{2 *}^{\mathrm{mic}}\right)$

where the subscripts $k$ and $k+1$ represent the iteration number. $V_{j^{*}}^{\text {mic }}$ is uniform form of $V_{j *}^{\mathrm{mic}, 1}$ and $V_{j *}^{\mathrm{mic}, 2}$ in Eqs. (4) and (5), and $V_{2 *}^{\mathrm{mic}}$ denotes the prescribed volume of base material 2 in the micro models correspondingly. As can be observed 
from Eq. (24), the volume fraction of base material 2 is sustainable increase until the prescribed volume of base material 2 is satisfied. Then, the volume of base material 2 remains unchanged and the volume of base materials 1 continues to reduce which means the volume of base material 3 begins to increase. As previously mentioned, the material interchanging just occurs between two neighboring phases, i.e., the transition between base materials 1 and 3 is interchanged through changing into base material 2 firstly. The transition of base materials 2 and 3 in the micro models is executed according to the sensitivity number $\alpha_{i 2}^{\text {mic }}$ and the target volume of base material 3 for the next iteration. The target volume of base material 3 for the next iteration can be defined using the same way as base material 2. Therefore, the volume of base material 3 is sustainable increase until the prescribed volume of base material 3 is satisfied. The interchanging process is terminated when the volume constraints of all base materials are satisfied. In addition, when base material number $n$ is larger than 3 , a similar procedure can be applied to make adjustment between base materials.

\subsection{Filtering scheme}

In order to avoid the common numerical instabilities such as checkerboard pattern and mesh-dependency in topology optimization, filter scheme is employed here to average the elemental sensitivity number in macro model as [12]:

$\alpha_{m}^{m a c}=\frac{\sum_{q=1}^{M} \omega\left(r_{m q}\right) \alpha_{q}^{m a c}}{\sum_{q=1}^{M} \omega\left(r_{m q}\right)} \quad$ where $\omega\left(r_{m q}\right)=\max \left(r_{\min }-r_{m q}, 0\right)$

where $r_{\min }$ is the filter radius, and $a_{m}^{m a c}$ is the filtered sensitivity value of $m$-th element in macro model. $r_{m q}$ denotes the distance between the center of element $m$ and $q$. To improve the convergence of the proposed method, the sensitivity number can be further averaged with its historical information in last iteration step:

$\left(\alpha_{m}^{m a c}\right)^{k}=\frac{\left(\alpha_{m}^{m a c}\right)^{k}+\left(\alpha_{m}^{m a c}\right)^{k-1}}{2}$ 
Note that sensitivity numbers in the micro models $\alpha_{i j}^{m i c, 1}$ and $\alpha_{i j}^{m i c, 2}$ can also be filtered according to Eq. (25) with filter radius $r_{\min }^{m i c}$ and respectively averaged with their historical information in the same way.

\subsection{Numerical implementation}

Take $n=3$ as an example, the whole numerical implementation procedure for the concurrent topological design of composite macrostructures and the underlying multi-phase materials is outlined as follows:

Step 1: Set BESO parameters: the prescribed volumes of first phase and second phase in the macro model, $V_{1}^{\text {mac }}$ and $1-V_{1}^{\text {mac }}$; the prescribed volumes of base materials in two micro models, $V_{j}^{\mathrm{mic}, 1}$ and $V_{j}^{\mathrm{mic}, 2}(j=1,2,3)$; the evolutionary ratio ER and the filter radii $r_{\min }^{m a c}$ for the macro model and $r_{\min }^{m i c}$ for the micro models.

Step 2: Construct initial design schemes of the macrostructure and material RUCs in macro and micro models respectively.

Step 3: Carry out the FE analysis for two micro models based on the homogenization approach, and calculate the effective elasticity matrices according to Eqs. (11) and (12).

Step 4: Substitute the obtained homogenized materials properties into the macro model and carry out the FE analysis for the macrostructure.

Step 5: Calculate the sensitivity numbers $\alpha_{m}^{m a c}$ at the macro model and $\alpha_{i 1}^{m i c, 1}$ and $\alpha_{i 1}^{m i c, 2}$ at the micro models according to Eqs. (13-22). Calculate sensitivity numbers $\alpha_{i 2}^{m i c, 1}$ and $\alpha_{i 2}^{m i c, 2}$, if the target volumes of base materials 2 in two micro models have been satisfied.

Step 6: Process all calculated sensitivity numbers in both macro and micro models with filtering and history-averaging.

Step 7: Update elements according to the elemental sensitivities and target 
volumes for the next iteration in each model. If all volume constraints in the macro and micro models are satisfied and the objective function becomes convergent, go to step 8. Otherwise, go to step 3.

Step 8: Output final topology results. Stop.

\section{Numerical examples and discussions}

In this section, several $2 \mathrm{D}$ and $3 \mathrm{D}$ numerical examples are presented to demonstrate the validity of the proposed method for concurrent topology optimization of composite macrostructures and their multi-phase materials. The RUCs are discretized into $100 \times 100$ four-node quadrilateral (Q4) elements for the $2 \mathrm{D}$ numerical examples and $30 \times 30 \times 30$ brick elements for the $3 \mathrm{D}$ numerical examples. The filter radii for the micro models $r_{\min }^{\text {mic }}$ are equal to 7 and 2 in $2 \mathrm{D}$ and $3 \mathrm{D}$ examples, respectively. The elements in all 2D and 3D macrostructures are assigned with the dimensions $1 \times 1$ and $1 \times 1 \times 1$, respectively. The filter radii in $2 \mathrm{D}$ and $3 \mathrm{D}$ macro models are respectively equal to 6 and 3. All base materials used in two micro models have the same Poisson's ratio $\mu=0.3$.

\section{1. $2 D$ cantilever beam}

This example considers the concurrent topology optimization of a cantilever beam and the underlying multi-phase material microstructures. The dimension of the macrostructural design domain is $100 \times 60$, as shown in Fig. 2. Assume three-phase material design is addressed in both two micro models, and then there are totally six base materials which take part in the concurrent design process. The Young's moduli of three base materials in micro model 1 are $E_{1}^{1}=100, E_{2}^{1}=10$ and $E_{3}^{1}=1$, in micro model 2 are $E_{1}^{2}=0.1, E_{2}^{2}=0.01, E_{3}^{2}=0.001$, respectively. These materials are named as the first group of base materials in the following. The prescribed volume constraints of base materials 1,2 and 3 in each micro model are all set to be one third of the design domain. The target volumes of two phases in the macro model are set to 
be equal to each other which means volume usages for first and second phases both are $50 \%$.

Two initial design schemes are considered: initial design 1 shows the design domain is full with first phase material/composite except for four elements at the center of the design domain for second phase material/composite as illustrated in Fig. 3a; initial design 2 shows the design domain is full with first phase material/composite except for four corner elements for second phase material/composite as shown in Fig. 3b. Fig. 4 shows a summary of two concurrent designs of the $2 \mathrm{D}$ cantilever beam and material microstructures by considering two different initial designs. The two cases in Fig. 4 are, respectively: (1) the initial designs of both macro and micro models adopt initial design 1; (2) the initial designs of both macro and micro models adopt initial design 2. Optimal designs of two-phase macrostructures with the resulting compliance $C$ are shown in Fig. 4a, where first phase (composite 1) in both two cases is shown in black and second phase (composite 2) in light yellow. The optimal designs of two kinds of three-phase composite microstructures, together with its corresponding homogenized elasticity matrices, are given in Fig. 4b, where base materials 1 are shown in red, base materials 2 in green and base materials 3 in yellow.

As can be observed from Fig. 4, the optimal design of the macrostructure is independent on initial configurations, while the initial design of material RUC affects its final topology. Even so, the homogenized elasticity matrices of composite 1 and 2 in case (1) are respectively similar with the corresponding terms in case (2). This result is consistent with the common knowledge of the inverse homogenization design that different microstructures can possess the same physical property [16, 22]. What's more, the final compliances in two cases are approximately equal to each other. It should be mentioned that other design parameters such as the shape of base cell and filter radius all have influence on the resulted solution. The iteration processes of RUCs and volume fractions of base materials in case (1) are illustrated in Fig. 5. At the earlier stage of the whole optimization procedure, the volume fraction of base material 3 keeps to zero until the volume constraint of base material 2 is satisfied. 
Then, the volume fraction of base material 2 remains constant and the volume fraction of base material 3 is gradually increase. Finally, all base materials' volume fractions converge to the same final value which is equal to one third of the design domain. The iteration processes of objective compliances under two cases, together with the volume fraction of first phase and the macrostructure in case (1) are given in Fig. 6. It is seen that the histories of the objective compliance of case (1) and (2) are very similar, and both of them become convergent after 68 iterations.

\section{2. $2 D M B B$ beam}

The second example is to design the topology of a 2D MBB beam and its multi-phase materials concurrently. As shown in Fig. 7, the geometrical dimension of the macro design domain is $200 \times 50$. Because of the symmetry, only right half of the MBB beam is considered in FE analysis of the beam. Firstly, assume three-phase composite design is addressed in both two micro models and the Young's moduli of all used base materials are exactly the same as section 5.1. In addition, the volume constraints in both micro and macro models are the same as section 5.1 as well. To start the concurrent optimization procedure, initial design 1 shown in Fig. 3(a) is adopted for both two micro models, and the macrostructure is full with first phase. The summary of optimization results is illustrated in Fig. 8, as can be observed, microstructures of the 2D MBB beam are totally anisotropic which is differ from the results in section 5.1. It indicates the characteristic that constructing with anisotropic composite microstructures result the macro $2 \mathrm{D} \mathrm{MBB}$ beam with the minimum compliance. The whole concurrent design procedure is convergence after 69 iterations.

In order to investigate the effect of the degree of inhomogeneity of the homogeneous base materials on final results, second group of base materials with different elastic contrast compared with the first group are considered in this example. In this group, Young's moduli of three base materials in micro model 1 are $E_{1}^{1}=4$, $E_{2}^{1}=2$ and $E_{3}^{1}=1$, in micro model 2 are $E_{1}^{2}=0.5, E_{2}^{2}=0.25, E_{2}^{3}=0.125$, 
respectively. The elastic contrasts of two neighboring base materials are equal to 2 while the volume constraints in micro and macro models are also the same as before. The summary of final optimization results is shown in Fig. 9. It is seen that the final results of both macrostructure and material microstructures are distributed in different pattern comparing with those in Fig. 8, which is reasonable because the load-bearing abilities of base materials are changed. The whole concurrent design procedure is convergence after 64 iterations.

To further demonstrate the proposed topology optimization method in concurrent design of macrostructures and the underlying multi-phase materials, each phase in the macrostructure composes of four base materials is considered here. The Young's moduli of four base materials in micro model 1 are $E_{1}^{1}=16, E_{2}^{1}=8, E_{3}^{1}=4$ and $E_{4}^{1}=2$, in micro model 2 are $E_{1}^{2}=1, E_{2}^{2}=0.5, E_{2}^{3}=0.25$ and $E_{4}^{3}=0.125$, respectively. There are totally eight base materials which take part in the concurrent design process in this case. In the micro models, each base material is isovolumetric distributed in the design domain which means the material volume fractions of four base materials all are specified as $25 \%$. The volume usages for first and second phase in the macro model are equal to each other as well. After 80 iterations, the final designs of this case are obtained as shown in Fig. 10. The optimal design of the macrostructure is shown in Fig. 10 (a), where the second phase of macrostructure is shown in light blue. In Fig. 10 (b), the base materials 1, 2, 3 and 4 in each micro model are respectively shown in dark blue (darkest region), light blue, yellow and red. The two four-phase composites are also anisotropic as can be observed with their elasticity matrices. Fig. 11 illustrates the iteration processes of the compliance and volume fractions of this case.

\section{3. $3 D$ cantilever beam}

In this example, the concurrent design of a $3 \mathrm{D}$ cantilever beam and the underlying multi-phase materials is considered. The design domain of macrostructure is $60 \times 20 \times 4$, as shown in Fig. 12. The left edge of the macrostructure is fixed, and a 
unit concentrated load is applied at the middle point of right blow edge. The first group base materials given in section 5.1 are employed and the volume fractions of base materials 1, 2 and 3 are respectively prescribed as 50\%, 30\% and 20\% in each micro model. In macro model, the volume fraction of first phase in the macrostructure is set to be $50 \%$. To start the concurrent optimization procedure, the initial design given in Fig. $3 c$ is adopted both for macro and micro models. Fig. 3c shows that the design domain is full with first phase material/composite except eight central elements for second phase material/composite. The optimal design of the macrostructure is given in Fig. 13, where the first phase of the macrostructure is shown in green and the second phase in blue. Note that the topology of the first phase demonstrated in Fig. $13 \mathrm{~b}$ is similar with the traditional topological design of monoscale structure. The final macrostructural compliance is $C=0.144$. Optimal designs of microstructures of the three-phase composites 1 and 2, together with the corresponding homogenized elasticity matrices are respectively given in Fig. 14 and Fig. 15, where the base materials 1,2 and 3 are respectively shown in red, blue and green. As can be observed, the hardest base materials are mainly distributed in $x$-direction and $y$-direction, and the approximate laminated plate structures are generated. What's more, the whole concurrent design process of the $3 \mathrm{D}$ cantilever beam is convergence after only 44 iterations.

\section{4. $3 D \mathrm{MBB}$ beam}

In the last example, the concurrent topological design of a 3D MBB beam and their multi-material microstructures subjects to multiple external forces is given. The design domain of the macrostructure is $80 \times 20 \times 12$, as shown in Fig. 16. Here, the macrostructure has four unit concentrated force at the top face. The first group base materials formulated in section 5.1 are considered here, and the material usages of base materials 1, 2 and 3 in each micro model are restricted to be $30 \%, 40 \%$ and $30 \%$ of the design domain, respectively. Volume constraint of first phase in macro model is set to $30 \%$. To start the concurrent optimization procedure, the initial design scheme 
illustrated in Fig. 3c is adopted for the macrostructure and material RUCs. After 71 iterations, all base materials' target volumes are well satisfied and the macrostructural compliance converges to $C=1.653$. The optimal design of the macrostructure is given in Fig. 17, and the generated first phase of the macrostructure is comparable with the design of monoscale macrostructure. The optimal material microstructural topologies of composites 1 and 2 are respectively illustrated in Fig. 18 and Fig. 19, where also showcase the spatial distributions of each base materials as well as the homogenized elasticity matrices. It is seen that the topological structures of composites 1 and 2 are totally different and the distinctive interfaces between different phases in both macro and micro structures are emerged.

\section{Conclusions}

In this paper, we have extended the concurrent topology optimization for the design of composite structures and the underlying multi-phase material microstructures. The composite configurations of the macrostructure and the material RUCs are optimized concurrently such that the resulting macrostructure has the minimum compliance. The $2 \mathrm{D}$ and $3 \mathrm{D}$ numerical results have shown that the presented method can provide efficient composite macrostructures with underlying three or more phases material microstructures in terms of stiffness. The generated interfaces between different base materials or phases are clear and distinctive which facilitates the manufacturing requirements. The presented approach has shown high stability during the design procedure and requires a small number of iterations for convergence. It is also worth mentioning that the presented methodology can be extended to the concurrent topological design of composite structures and multi-functional materials, which are absolutely composed of two or more base materials, for different objectives or multi-objective functions.

\section{Acknowledgments}

This work was supported by the National Science Foundation of China (11472101), Postdoctoral Science Foundation of China (2013M531780) and State Key Program of 
National Natural Science of China (61232014).

\section{References}

[1] Bendsøe MP, Sigmund O. Topology optimization: theory, methods and applications. Berlin: Springer-Verlag; 2003.

[2] Zhu JH, Zhang WH, Xia L. Topology Optimization in Aircraft and Aerospace Structures Design. Arch Computat Methods Eng DOI 10.1007/s11831-015-9151-2

[3] Bendsøe MP, Kikuchi N. Generating optimal topologies in structural design using a homogenization method. Comput Methods Appl Mech Eng 1988; 71:197-224.

[4] Bendsøe MP. Optimal shape design as a material distribution problem. Struct Optim 1989;1: 193-202.

[5] Zhou M, Rozvany GIN. The COC algorithm, part II: topological, geometry and generalized shape optimization. Comput Methods Appl Mech Eng 1991;89: 197-224.

[6] Wang MY, Wang X, Guo D. A level set method for structural topology optimization. Comput Methods Appl Mech Eng 2003;192:227-46.

[7] Allaire G, Jouve F, Toader AM. Structural optimization using sensitivity analysis and a level-set method, J Comput Phys. 2004;194 (1):363-393.

[8] Xie YM, Steven GP. Simple evolutionary procedure for structural optimization. Comput Struct 1993;49(5):885-96.

[9] Xie YM, Steven GP. Evolutionary Structural Optimization, London: Springer; 1997.

[10] Huang X, Xie YM, Burry MC. A new algorithm for bi-directional evolutionary structural optimization. JSME Int J, Ser C 2007;49(4):1091-9.

[11] Huang X, Xie YM. Evolutionary topology optimization of continuum structures: methods and applications. Chichester: John Wiley \& Sons; 2010.

[12] Sigmund O, Maute K, Topology optimization approaches - a comparative review, Struct Multidiscip Optim 2013;48(6):1031-1055.

[13] Huang X, Xie YM. Convergent and mesh-independent solutions for the bidirectional evolutionary structural optimization method. Finite Elem Anal Des 2007;43(14):1039-49.

[14] Huang X, Xie YM. Optimal design of periodic structures using evolutionary optimization. Struct Multi Optim 2008;36:597-609.

[15] Huang X, Zuo ZH, Xie YM. Evolutionary topology optimization of vibrating continuum structures for natural frequencies. Comput Struct 2010;88:357-64.

[16] Andreassen E, Andreasen CS. How to determine composite materials Properties using numerical homogenization. Comput Mater Sci 2014;83:488-495.

[17] Sigmund O. Materials with prescribed constitutive parameters: an inverse homogenization problem. Int J Solids Struct 1994;31(17):2313-29.

[18] Sigmund O. Tailoring materials with prescribed elastic properties. Mech Mater $1995 ; 20: 351-68$.

[19] Zhou SW, Li Q. A microstructure diagram for know bounds in conductivity. J Mater Res 2008;(23):798-811.

[20] Guest James K, Prévost Jean H. Optimizing multifunctional materials: design of microstructures for maximized stiffness and permeability. Int $\mathrm{J}$ Solids Struct 2006;43(22-23):7028-47. 
[21] V.J. Challis, A.P. Roberts, A.H. Wilkins, Design of three dimensional isotropic microstructures for maximized stiffness and conductivity, Int. J. Solids Struct. 2008; 45(14-15):4130-4146.

[22] Wang Y, Luo Z, Zhang N, Kang Z, Topological shape optimization of micro-structure metamaterials using a level set method, Comput Mater Sci 2014; 87: 178-186.

[23] Huang X, Radman A, Xie YM. Topological design of microstructures of cellular materials for maximum bulk or shear modulus. Comput Mater Sci 2011;50: 1861-70.

[24] Huang X, Xie YM, Jia B, Li Q, Zhou SW. Evolutionary topology optimization of periodic composites for extremal magnetic permeability and electrical permittivity. Struct Multi Optim 2012;46:385-98.

[25] Zhang WH, Dai GM, Wang FW, Sun SP, Bassir HC. Using strain energy-based prediction of effective elastic properties in topology optimization of material microstructure. Acta Mech Sin 2007;23(1):77-89.

[26] Guest JK, Prévost JH. Design of maximum permeability material structures. Comput Methods Appl Mech Eng 2007;196:1006-17.

[27] Neto MA, Yu WB, Tang T, Leal R. Analysis and optimization of heterogeneous materials using the variational asymptotic method for unit cell homogenization. Compos Struct 2010;92:2946-2954.

[28] Xia L, Breitkopf P. Design of materials using topology optimization and energy-based homogenization approach in Matlab. Struct Multi Optim 2015.

[29] Rodrigues H, Guedes JM, Bendsøe MP. Hierarchical optimization of material and structure. Struct Multi Optim 2002;24(1):1-10.

[30] Coelho PG, Fernandes PR, Guedes JM, Rodrigues HC. A hierarchical model for concurrent material and topology optimization of three-dimensional structures. Struct Multi Optim 2008;35:107-15.

[31] Ferreira RTL, Rodrigues HC, Guedes JM, Hernandes JA. Hierarchical optimization of laminated fiber reinforced composites. Compos Struct 2014;107:246-259.

[32] Xia L, Breitkopf P. Concurrent topology optimization design of material and structure within $\mathrm{fe}^{2}$ nonlinear multiscale analysis framework. Comput Methods Appl Mech Eng 2014;278:524-542.

[33] Xia L, Breitkopf P. A reduced multiscale model for nonlinear structural topology optimization. Comput Methods Appl Mech Eng 2014;280:117-134.

[34] Xia L, Breitkopf P. Recent Advances on Topology Optimization of Multiscale Nonlinear Structures. Arch Comput Methods Eng 2016; doi: 10.1007/s11831-016-9170-7.

[35] Xia L, Breitkopf P. Multiscale structural topology optimization with an approximate constitutive model for local material microstructure. Comput Methods Appl Mech Eng 2015;286:147-167

[36] Yvonnet J, Gonzalez D, He QC. Numerically explicit potentials for the homogenization of nonlinear elastic heterogeneous materials. Comput Methods Appl Mech Eng 2009;198:2723-2737.

[37] Yan X, Huang X, Zha Y, Xie YM. Concurrent topology optimization of structures and their composite microstructures. Comput Struct 2014.

[38] Liu L, Yan J, Cheng GD. Optimum structure with homogeneous optimum trusslike material. Comput Struct 2008;86(13-14):1417-25. 
[39] Niu B, Yan J, Cheng GD. Optimum structure with homogeneous optimum cellular material for maximum fundamental frequency. 2009;39:115-132.

[40] Zuo ZH, Huang XD, Rong JH, Xie YM. Multi-scale design of composite materials and structures for maximum natural frequencies. Mater Des 2013;51: 1023-34.

[41] de Kruijf N, Zhou SW, Li Q, Mai YW. Topological design of structures and composite materials with multiobjectives. Int J Solids Struct 2007;44:7092-109.

[42] Deng JD, Yan J, Cheng GD. Multi-objective concurrent topology optimization of thermoelastic structures composed of homogeneous porous material. Struct Multi Optim 2013;47(4):583-97.

[43] Yan XL, Huang X, Sun GY, Xie YM. Two-scale optimal design of structures with thermal insulation materials. Compos Struct 2015;120:358-365.

[44] Guo X, Zhao XF, Zhang WS, Yan J, Sun GM. Multi-scale robust design and optimization considering load uncertainties. Comput Methods Appl Mech Eng 2015; 283:994-1009.

[45] Xu B, Xie YM. Concurrent design of composite macrostructure and cellular microstructure under random excitations. Compos Struct 2015;123:65-77.

[46] Gibson RF. A review of recent research on mechanics of multifunctional composite materials and structures. Compos Struct 2010;92:2793-2810.

[47] Bendsøe MP, Sigmund O. Material interpolation schemes in topology optimization, Arch Appl Mech.1999; 69: 635-654.

[48] Gibiansky. LV, Sigmund O. Multiphase composites with extremal bulk modulus. J Mech Phys Solids 2000;48:461-498.

[49] Sigmund O. Design of multiphysics actuators using topology optimization. Part II: two-material structures, Comput. Methods Appl. Mech. Eng. 2001;190:6605-6627.

[50] Blasques José Pedro, Stolpe Mathias. Multi-material topology optimization of laminated composite beam cross sections. Compos Struct 2012;94(11):3278-89.

[51] Blasques José Pedro. Multi-material topology optimization of laminated composite beams with eigenfrequency constraints. Compos Struct 2014;111:45-55.

[52] Wang MY, Wang XM, 'Color' level sets: a multiphase method for structural topology optimization with multiple materials, Comput Methods Appl Mech Engrg 2004; 469-496.

[53] Z. Luo, L.Y. Tong, J. Luo, M. Wang, Design of piezoelectric actuators using a multiphase level set method of piecewise constants, J. Comput. Phys. 228 (2009) 2643-2659.

[54] Wei P, Wang MY, Piecewise constant level set method for structural topology optimization, Internat J Numer Methods Engrg 2009;78: 379-402.

[55] Guo X, Zhang WS, Zhong WL. Stress-related topology optimization of continuum structures involving multi-phase materials. Comput Methods Appl Mech Engrg 2014; 268:632-655.

[56] Huang X, Xie YM. Bi-directional evolutionary topology optimization of continuum structures with one or multiple materials. Comput Mech 2009;43(3):393-401.

[57] Xu B, Jiang JS, Xie YM. Concurrent design of composite macrostructure and multi-phase material microstructure for minimum dynamic compliance. Compos Struct 2015;128:221-233.

[58] Radman A, Huang X, Xie YM. Topological design of microstructures of multi-phase materials for maximum stiffness or thermal conductivity. Comput Mater Sci 2014;91:266-273.

[59] Hassani B, Hinton E. A review of homogenization and topology optimization I 
homogenization theory for media with periodic structure. Comput Struct 1998;69:707-17.

[60] Hassani B, Hinton E. A review of homogenization and topology optimization II analytical and numerical solution of homogenization equations. Comput Struct 1998;69:719-38.

\section{Figures:}

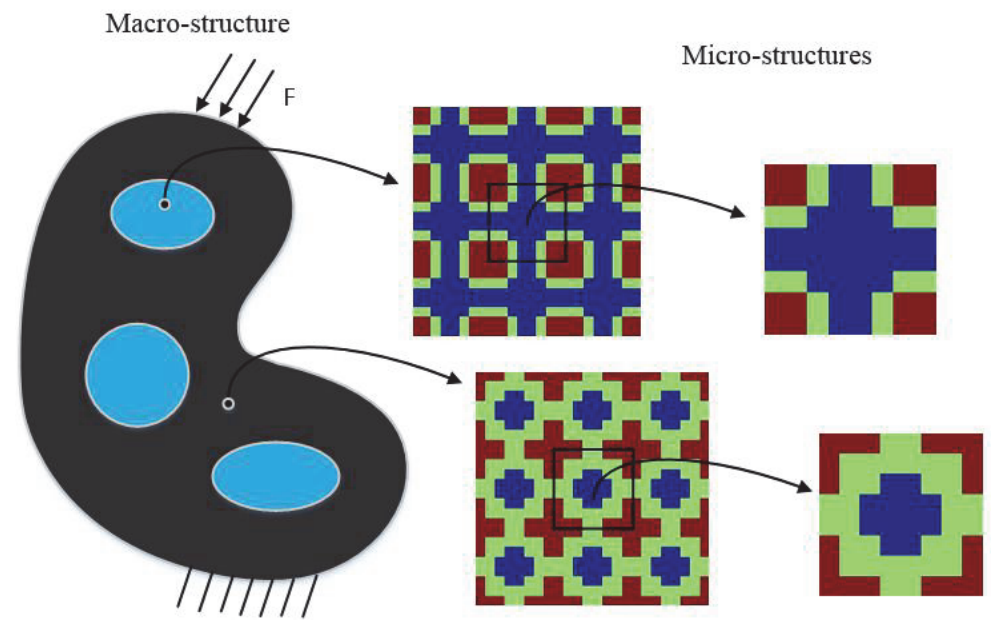

Fig. 1. An arbitrary macrostructure composed of periodic microstructures.

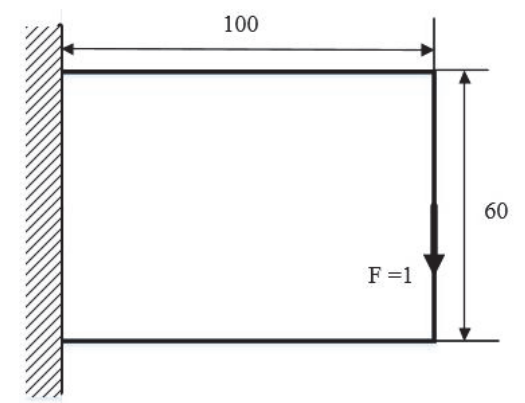

Fig. 2. Geometrical dimension and boundary conditions for a 2D cantilever beam.

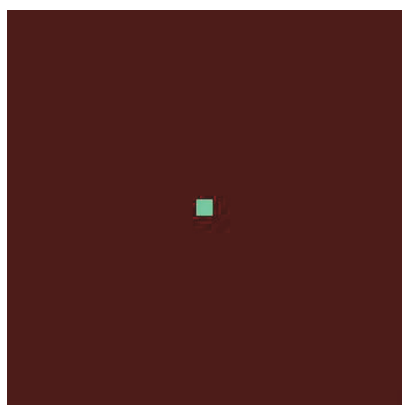

(a)

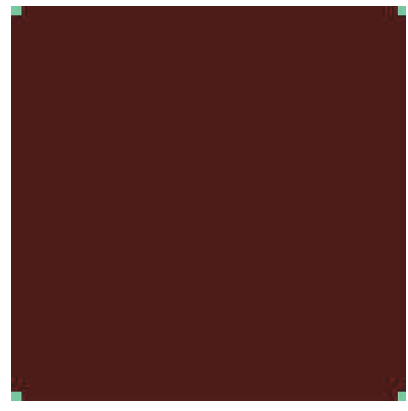

(b)

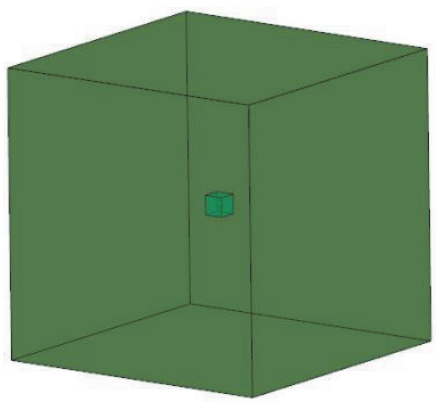

(c)

Fig. 3. Initial designs (a) initial design 1 of 2D cases; (b) initial design 2 of 2D cases; (c) 3D cases. 
(a)

(1)

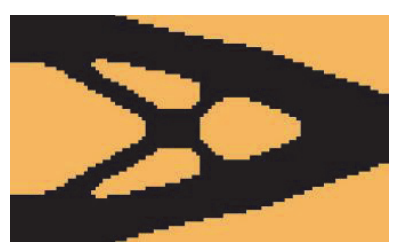

(2)

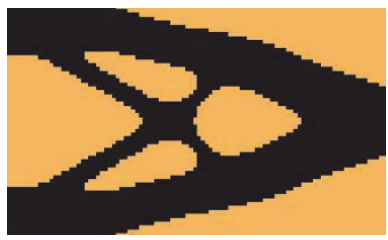

(b)

Case

Composite 1 (RUC, $3 \times 3$ unit cells and Composite 2 (RUC, $3 \times 3$ unit cells and elasticity matrix)

(1)
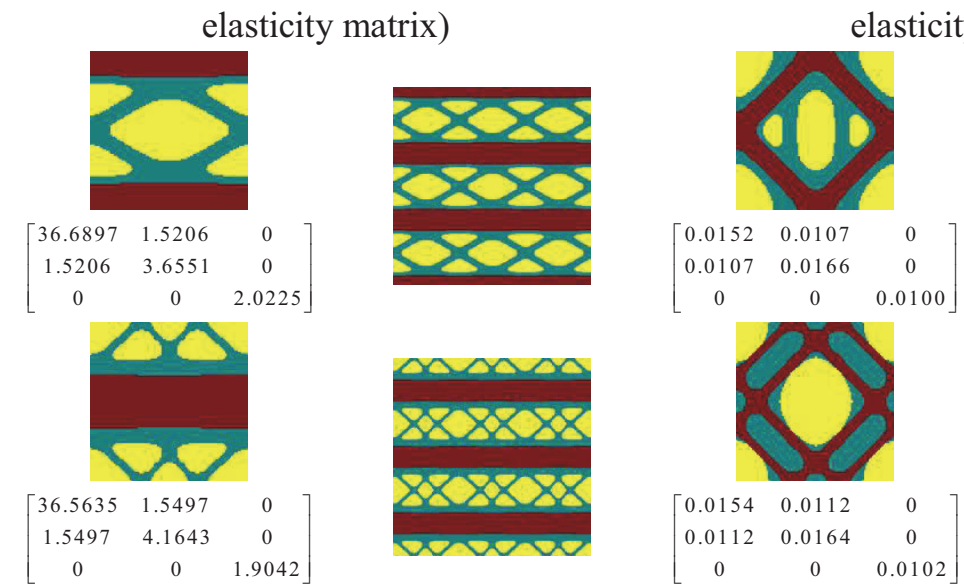
elasticity matrix)

\section{(2)}
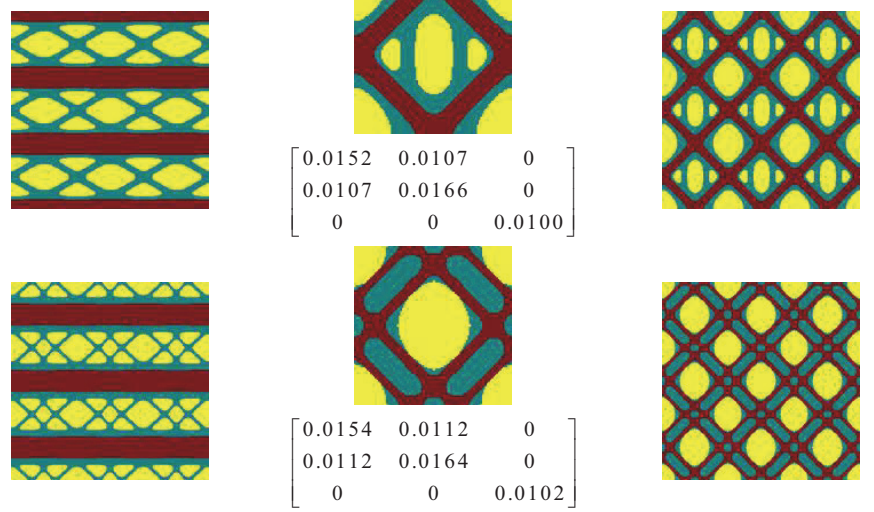

Fig. 4. Summary of two concurrent designs of the 2D cantilever beam considering two different initial configurations: (a) macrostructures and compliances; (b) microstructures and elasticity matrices.

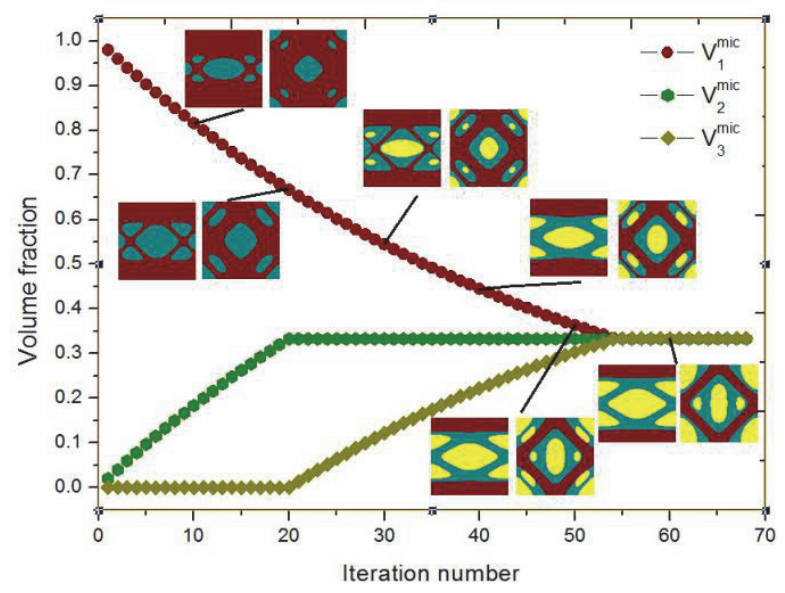

Fig. 5. Iteration processes of microstructural topology and volume fractions. 


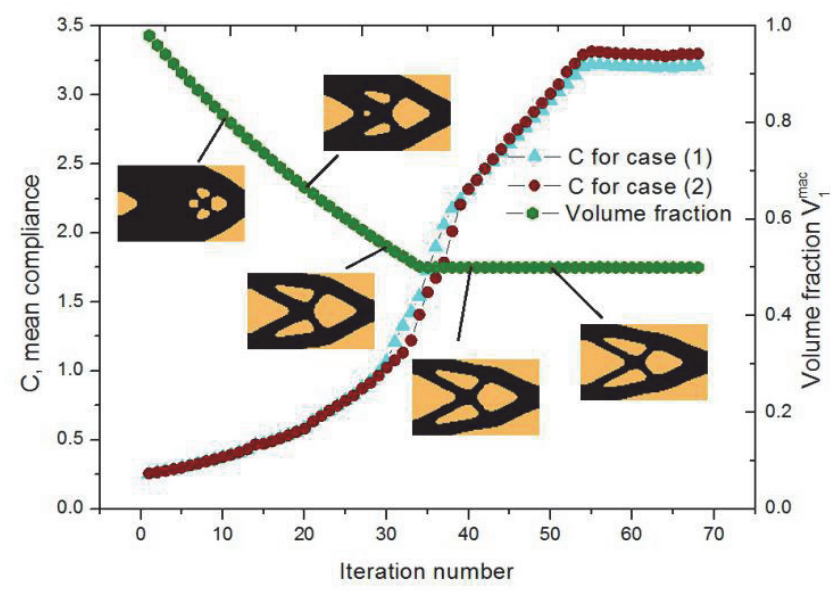

Fig. 6. Iteration processes of compliances, volume fraction and macrostructural topology.

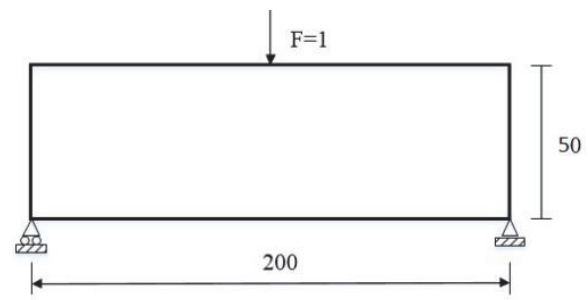

Fig. 7. Geometrical dimension and boundary conditions for a 2D MBB beam.

(a)

Macrostructure

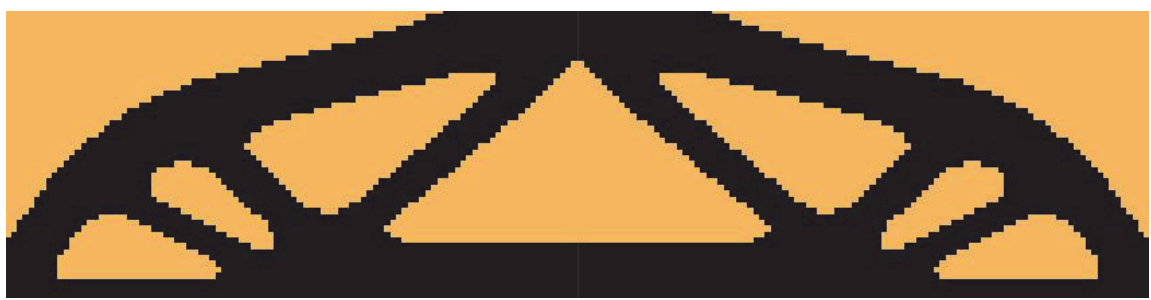

Mean compliance

(b)

\section{Composite 1}

RUC and elasticity matrix

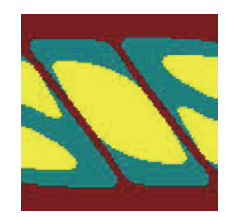

$\left[\begin{array}{lll}31.0421 & 3.4318 & 2.1746\end{array}\right]$ $\begin{array}{lll}3.4318 & 9.0327 & 3.9556\end{array}$ $\left[\begin{array}{lll}2.1746 & 3.9556 & 4.2094\end{array}\right]$
$3 \times 3$ unit cells

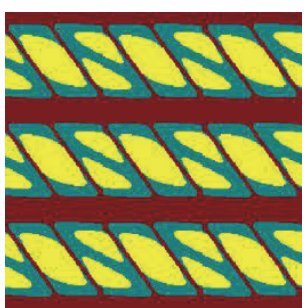

Composite 2

RUC and elasticity matrix
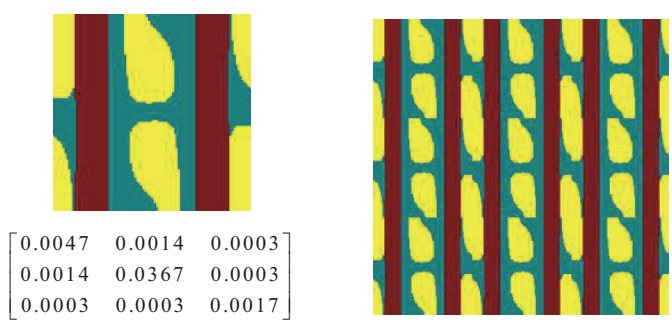

Fig. 8. Summary of final designs for the $2 \mathrm{D}$ MBB beam with first group base materials. (a) macrostructure and compliance; (b) microstructures and elasticity matrices. 
(a)

Macrostructure

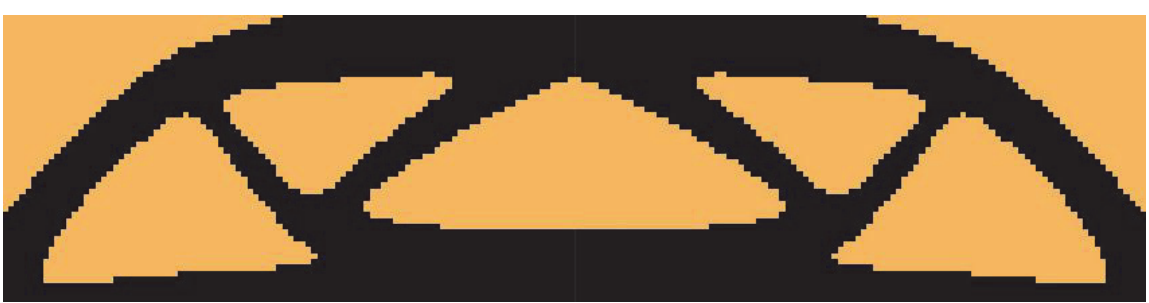

Mean compliance

32.895

(b)

\section{Composite 1}

RUC and elasticity matrix

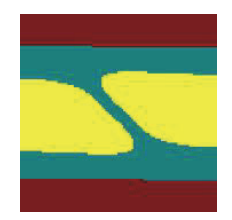

$\left[\begin{array}{lll}2.4878 & 0.5725 & 0.0081\end{array}\right]$ $\left[\begin{array}{lll}0.5725 & 1.8956 & 0.0081 \\ 0.0081 & 0.0081 & 0.6690\end{array}\right]$

$\begin{array}{lll}0.0081 & 0.0081 & 0.6690\end{array}$
$3 \times 3$ unit cells

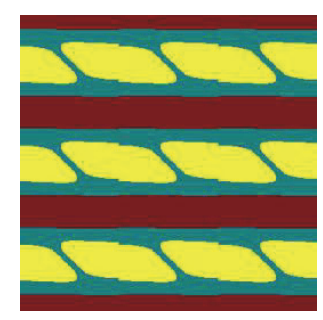

Composite 2

RUC and elasticity matrix

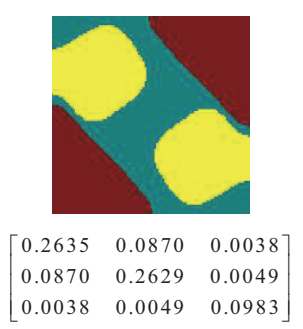

$3 \times 3$ unit cells

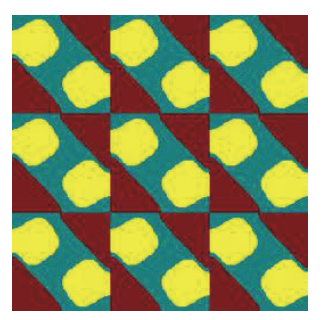

Fig. 9. Summary of final designs for the $2 \mathrm{D}$ MBB beam with second group base materials. (a) macrostructure and compliance; (b) microstructures and elasticity matrices.

(a)

Macrostructure

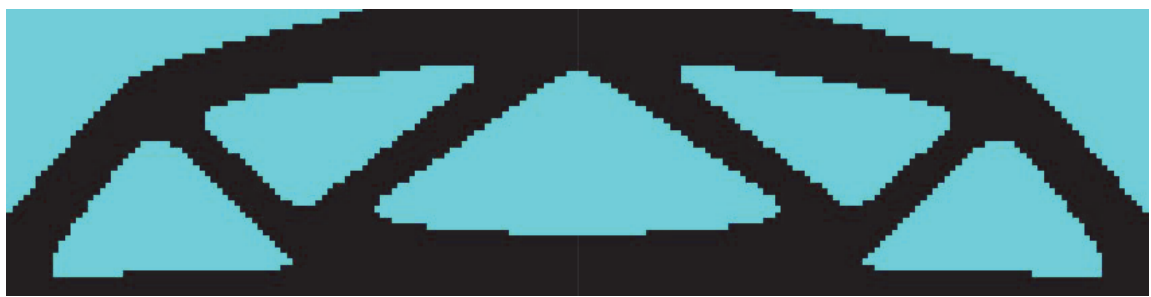

Mean compliance

12.201

(b)

Composite 1

RUC and elasticity matrix

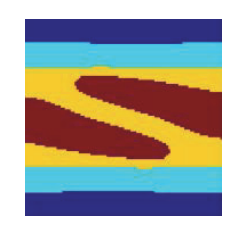

$\left[\begin{array}{lll}7.8779 & 1.4243 & 0.0437\end{array}\right]$ $\begin{array}{lll}1.4243 & 4.6983 & 0.0121\end{array}$ $\left[\begin{array}{lll}0.0437 & 0.0121 & 1.6716\end{array}\right]$
$3 \times 3$ unit cells

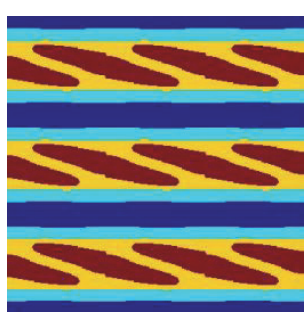

Composite 2

RUC and elasticity matrix

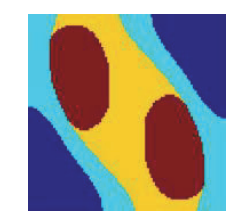

$\left[\begin{array}{lll}0.3461 & 0.1173 & 0.0120\end{array}\right]$ $\left.\begin{array}{lll}0.1173 & 0.3978 & 0.0154 \\ 0.0120 & 0.0154 & 0.1304\end{array}\right]$
$3 \times 3$ unit cells

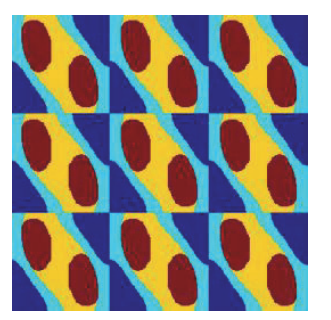

Fig. 10. Summary of final designs for the $2 \mathrm{D}$ MBB beam with eight base materials. (a) macrostructure and compliance; (b) microstructures and elasticity matrices. 


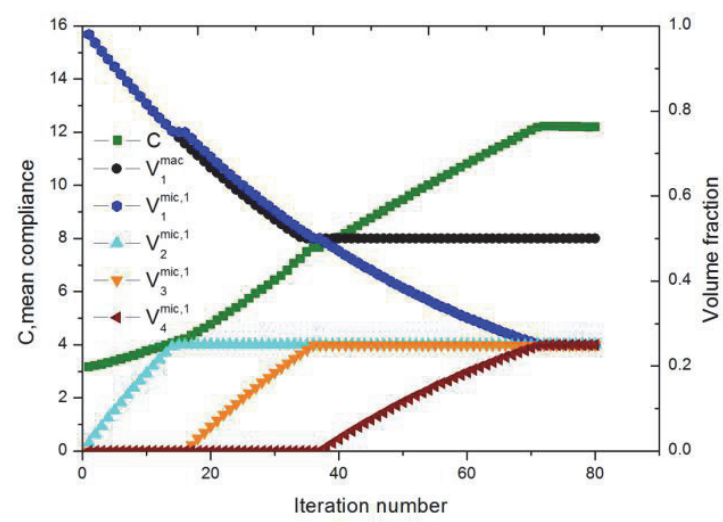

Fig. 11. Iteration processes of compliance and volume fractions.

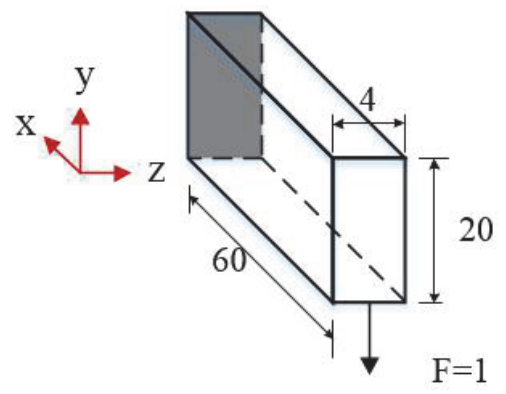

Fig. 12. Geometrical dimension and boundary conditions for a 3D cantilever beam.

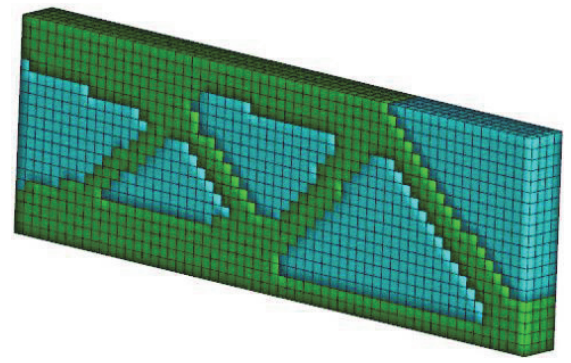

(a) Macrostructure

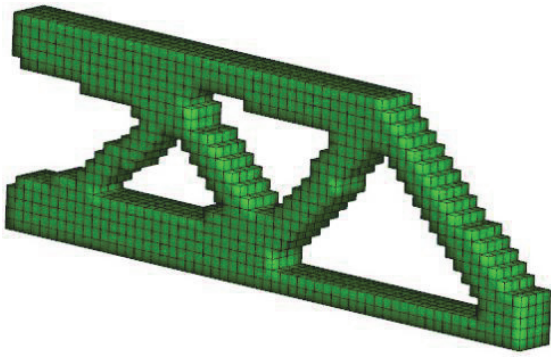

(b) Macrostructure (first phase only)

Fig. 13. Optimal design of macrostructure for the 3D cantilever beam. 


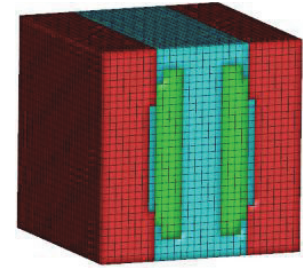

(a)

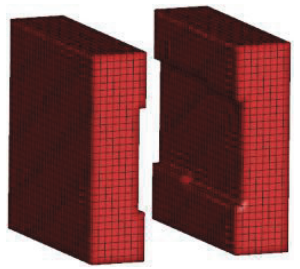

(b)

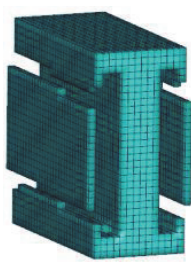

(c)

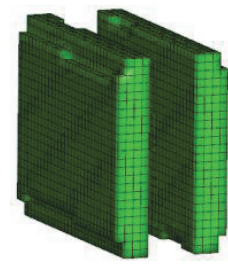

(d)

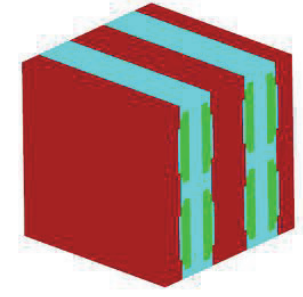

(e)

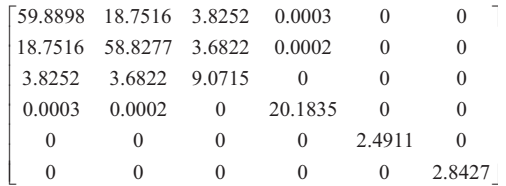

(f)

Fig. 14. Optimized material microstructures of composite 1 for the 3D cantilever beam; (a) micro RUC (b) base material 1 only; (c) base material 2 only; (d) base material 3 only; (e) $2 \times 2 \times 2$ unit cells; (f) homogenization elasticity matrix.

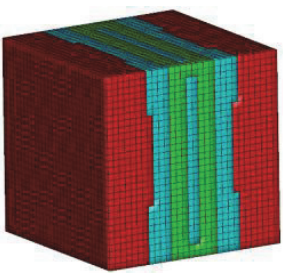

(a)

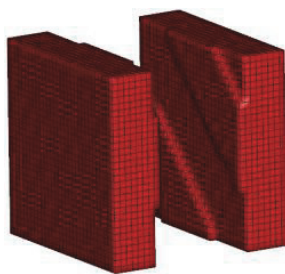

(b)

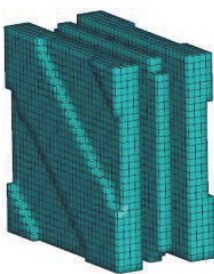

(c)

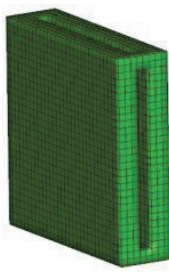

(d)

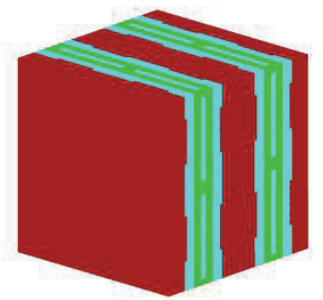

(e)

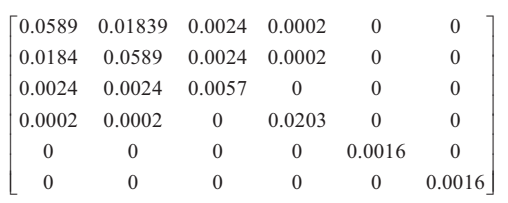

(f)

Fig. 15. Optimized material microstructures of composite 2 for the 3D cantilever beam; (a) micro RUC (b) base material 1 only; (c) base material 2 only; (d) base material 3 only; (e) $2 \times 2 \times 2$ unit cells; (f) homogenization elasticity matrix.

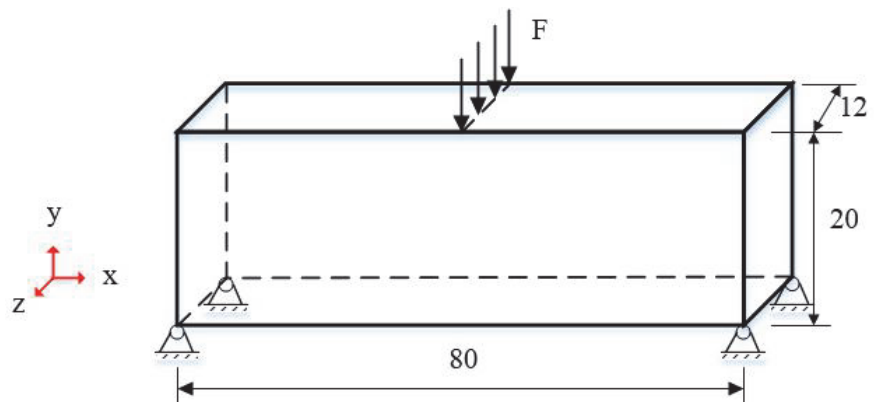

Fig. 16. Geometrical dimension and boundary conditions for a 3D MBB beam. 


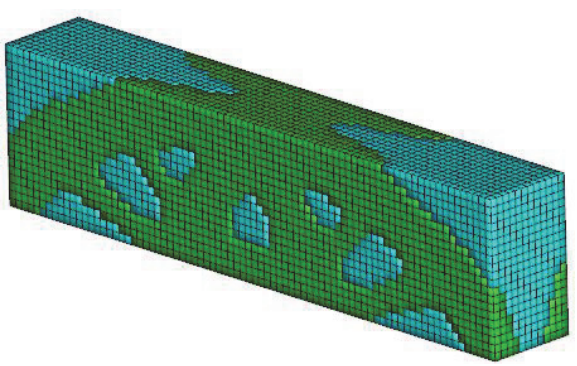

(a) Macrostructure

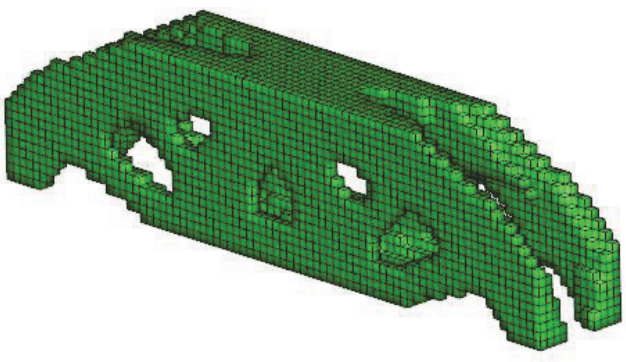

(b) Macrostructure (first phase only)

Fig. 17. Optimal design of macrostructure for the 3D MBB beam.

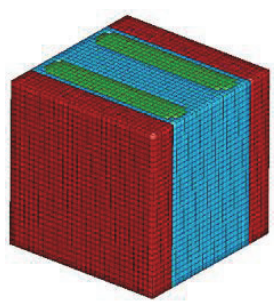

(a)

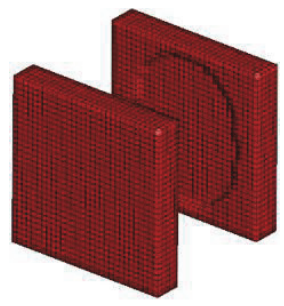

(b)

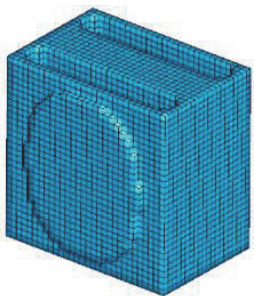

(c)

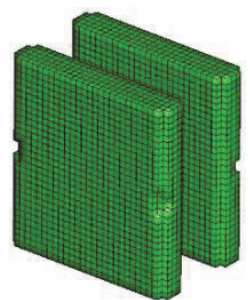

(d)

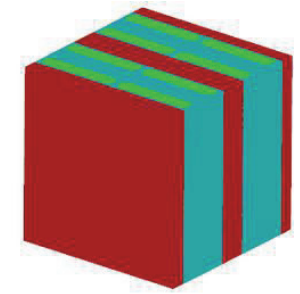

(e)

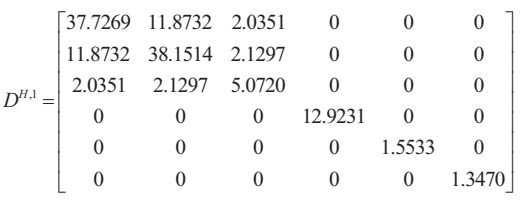

(f)

Fig. 18. Optimized material microstructures of composite 1 for the 3D MBB beam; (a) micro RUC (b) base material 1 only; (c) base material 2 only; (d) base material 3 only; (e) $2 \times 2 \times 2$ unit cells; (f) homogenization elasticity matrix. 


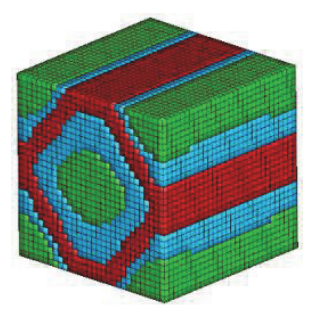

(a)

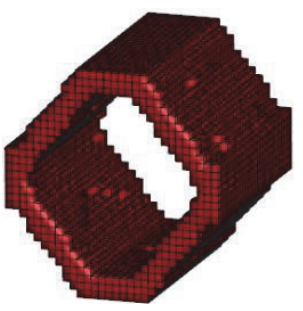

(b)

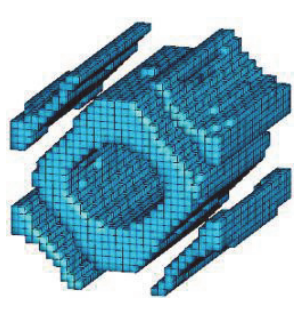

(c)

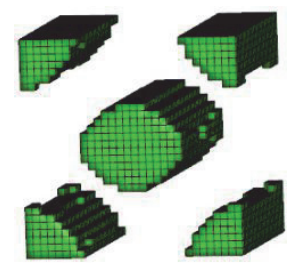

(d)

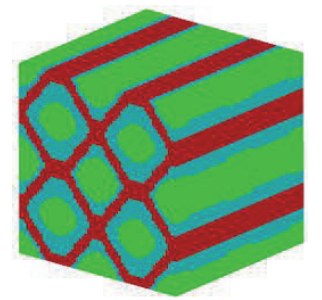

(e)

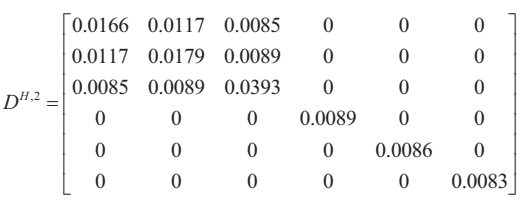

(f)

Fig. 19. Optimized material microstructures of composite 2 for the 3D MBB beam; (a) micro RUC (b) base material 1 only; (c) base material 2 only; (d) base material 3 only; (e) $2 \times 2 \times 2$ unit cells (f) homogenization elasticity matrix. 